\title{
Hepatic artery infusion pump for nasopharyngeal carcinoma with liver metastasis
}

\author{
Changli Peng ${ }^{1}$. Chunhui Zhou ${ }^{1}$ Gang $\mathrm{Li}^{1} \cdot$ Haiping $\mathrm{Li}^{1} \cdot$ Liangrong Shi ${ }^{1} \mathbb{C}$
}

Received: 23 August 2019 / Accepted: 12 December 2019 / Published online: 20 December 2019

(c) The Author(s) 2019

\begin{abstract}
To evaluate the benefits and risks of hepatic artery infusion (HAI) gemcitabine and floxuridine (FUDR) in patients with nasopharyngeal carcinoma liver metastases. HAI catheter systems were implanted under the guide of digital subtract angiography (DSA) in 16 patients with unresectable nasopharyngeal carcinoma liver metastases. HAI gemcitabine and FUDR in combination with radiotherapy and systemic chemotherapy were delivered. Disease control rate (DCR) of intrahepatic lesions is $100 \%$, objective response rate (ORR) of intrahepatic lesions is $87.5 \%$, including 4 patients (25\%) with complete response (CR), 10 patients (62.5\%) with partial response (PR) and 2 patients (12.5\%) with stable disease (SD). The median overall survival (mOS) was 30 months. There was no significant difference between patients with $<9$ intrahepatic lesions and patients with $\geq 9$ intrahepatic lesions ( 31 months vs. 24 months, $P=0.562$ ). Patients without extrahepatic metastases has longer survival than patients with extrahepatic metastases ( 31 months vs. 17 months, $P=0.005$ ). In all 72 cycles of HAI, the main grade $3 / 4$ toxicities related to HAI include: leukopenia occur in 8 cycles $(11.1 \%)$, thrombocytopenia in 5 cycles $(6.9 \%)$, AST/ALT elevation in 12 cycles (16.7). Catheter related complications occurred in 2 patients (12.5\%). HAI gemcitabine and FUDR is effective to improve DCR of intrahepatic lesions and prolong mOS for patients with nasopharyngeal carcinoma liver metastases, and is associated with a relative low rate of toxicity.
\end{abstract}

Keywords Hepatic artery infusion $\cdot$ Nasopharyngeal carcinoma $\cdot$ Liver metastasis

\section{Introduction}

Nasopharyngeal carcinoma (NPC) is a common head and neck malignancy, with a high prevalence in South-East Asia, particularly in southern China [1]. It is estimated that in China, NPC accounts for about $1.34 \%$ of all new cancer cases per year and $1.03 \%$ of all cancer deaths per year, with a prevalence of 3.16/100,000 and 1.53/100,00 respectively [2]. Unlike other head and neck cancers, NPC presents with high incidence of locoregional recurrence or distant metastases [3], which is considered as the predominant cause of mortality [4]. The most common metastatic sites are bone, liver and lung [5]. Liver metastases of NPC often present as multifocal nodules, and unfortunately has worse prognosis compared with metastasis to bone or to lung, with

Liangrong Shi

shiliangrong_xy@163.com

1 Radiological Intervention Center, Department of Radiology, Xiangya Hospital, Central South University, 87 Xiangya Road, Changsha 410008, Hunan, China median overall survival of 3-5 months [6, 7]. According to NCCN guidelines, treatment options for metastatic NPC include Clinical trials (preferred), Platinum-based combination chemotherapy or concurrent chemo/radiotherapy [8], but the clinical outcome is still limited. Thus how to improve the prognosis of NPC patients with liver metastasis remains a big challenge.

It is reported that perioperative hepatic arterial infusion pump chemotherapy is associated with longer survival after resection of colorectal liver metastases [9]. Previous work by our team showed that initial hepatic artery infusion (HAI) and systemic chemotherapy is helpful for colorectal cancer patients with liver metastases to obtain a high resection rate [10]. Hepatic-directed therapy is recommended for some liver-predominant disease in patients with neuroendocrine tumors of the pancreas [11]. Here, we use this HAI regime in NPC patients with liver-predominant metastases, aiming to verify its effectiveness and safety. 


\section{Patients and methods}

\section{Patients selection}

From January 2011 to December 2017, we treated 16 consecutive patients with NPC liver-predominant metastases. The following clinical data were collected: age, gender, performance status, UICC stage, pathological type, type of systemic chemotherapy, volume proportion of involved liver, number of intrahepatic lesions, status of portal vein thrombus, extrahepatic metastases, Child-Pugh class of liver function, objective response status of intrahepatic lesions (assessed with CT-enhanced scan according to RECIST criteria), survival in months from time of catheter implantation. The treatment protocol for each patient was discussed and determined by a multi-discipline treatment (MDT) in our hospital, which includes medical oncologist, otolaryngologist, radiologist and interventional specialist. Before the initial treatment, an informed consensus was achieved, which was approved by the Ethics Committee of our hospital.

\section{HAI catheter system implantation}

The infusion catheter and injection port (Celsite, B. Braun, Chasseneuil, France) was implanted as previously described by our team and other authors $[10,12,13]$. The key points of the technique include: (i) Computed tomography angiography before operation to assess the anatomy of hepatic artery and indication for HAI therapy; (ii) Under the guide of digital subtraction angiography (DSA), the Seldinger technique was used to establish access to right femoral artery. (iii) Angiography of celiac trunk and superior mesenteric artery was performed to confirm the anatomy of hepatic artery; (iv) Embolization of the gastro-duodenal artery (GDA), right gastric artery, and, if necessary, left gas-tric artery or dorsal pancreatic artery with metal coils (Tornade, Cook, Bloomington, IL, USA) to prevent extra-hepatic infusion of chemotherapeutic agents and resulting gastro-duodenal injury; (v) Side-hole infusion catheter was inserted in GDA or peripheral branch of hepatic artery, followed by the corresponding vessels embolized using the same coils as mentioned above to fix the infusion catheter, with the side-hole positioned in the common hepatic artery. Thus the chemotherapeutic agents could infuse the entire liver from the side-hole; (vi) the proximal end of the infusion catheter was connected to an injection port and the device was implanted in a subcutaneous pocket in the right inner thigh. After the administration of chemotherapeutic agents, the implanted port and indwelling catheter system were flushed and filled with $2 \mathrm{~mL}$ of heparin solution (1000 IU/mL).

\section{HAI therapy and systemic chemotherapy}

All patients received a 3-week cycle of HAI the next day after catheter implantation. The HAI therapy was performed on day 1, 8: Gemcitabine $1 \mathrm{~g} / \mathrm{m}^{2}$ for $30 \mathrm{~min}$, followed by a blended solution which comprised floxuridine (FUDR) at $0.15 \mathrm{mg} / \mathrm{kg} /$ day, dexamethasone (DXM) at $1 \mathrm{mg} / \mathrm{m}^{2} /$ day, low molecular heparin $3200 \mathrm{U}$ and saline, lasted for 7 days continuously. This type of HAI regime was accomplished by a 14-day infusor (Baxter). Standard treatment of NPC, including radiotherapy and chemotherapy (induction chemotherapy, concurrent chemotherapy and adjuvant chemotherapy) was performed as necessary. Dose adjustment was made in the event of toxicity, assessed according to National Cancer Institute-Common Terminology Criteria for Adverse Events (NCI-CTCAE) version 3.0. The HAI therapy was stopped if serious technical catheter-related problems, progression of intrahepatic disease or excessive toxicity occurred. Response to chemotherapy was assessed every 2 HAI cycles or when necessary by spiral-CT scan according to the RECIST criteria [14]. Epigastric pain prompted workup with an upper gastrointestinal endoscopy. If an ulcer or gastro-duodenitis was documented, HAI therapy was held for 1 month to allow healing and the dosage of FUDR and DXM was reduced by $50 \%$ in subsequent therapies.

\section{Statistical analysis}

The main endpoint of the study was objective response rate (ORR). Overall survival (OS) was defined as the time from the date of catheter implantation to the date of death or the date of the last follow-up, median survival time (mOS) was defined as the time from the date of catheter implantation to the date $50 \%$ of individuals is alive. The 3 -year survival rates were estimated by using the Life Table method. The survival analysis was performed by using the log-rank test.

\section{Results}

\section{Patients' baseline of characteristics}

Sixteen patients (13 males and 3 females) were included in the study, with the median age of 56yrs (30-78 years). All of them scored 0-2 in ECOG (Eastern Cooperative Oncology Group) score standard, staged II-IV in UICC (The Union of International Cancer Control) stage system. The pathology type of NPC was confirmed by biopsy, comprised Non-keratinized differentiated type $(\mathrm{n}=2)$ and Nonkeratinized undifferentiated type $(n=14)$. Systemic chemotherapy was performed in all patients, which included induction chemotherapy, concurrent chemotherapy and 
adjuvant chemotherapy. All of the baseline of characteristics are summarized in Table 1.

\section{Baseline information of hepatic metastases}

The status of hepatic metastases, including synchronous hepatic metastases at initial diagnosis of NPC, and disease progresses to hepatic metastases, was assessed by proportion of hepatic involvement, lobular involvement, number of hepatic lesions, existence of extrahepatic metastases, and baseline level of albumin. See Table 2. Notice that majority of patients presents bilobar involvement $(n=13$, $81.3 \%$ ), multifocal metastases ( 9 patients with intrahepatic lesions $\geq 9,56.2 \%$ ). No patient is indicated for metastases resection (diffused intrahepatic lesions or bilobar involvement).

Table 1 Baseline of characteristics $(n=16)$

\begin{tabular}{ll}
\hline Characteristics & $\mathrm{n}(\%)$ \\
\hline Age (year) & \\
Median & 56 \\
Range & $30-78$ \\
Gender & \\
Male & $13(81.3)$ \\
Female & $3(18.7)$ \\
Performance status & \\
0 & $5(31.25)$ \\
1 & $10(62.5)$ \\
2 & $1(6.25)$ \\
UICC stage & \\
II & $3(18.7)$ \\
III & $11(68.8)$ \\
IV & $2(12.5)$ \\
Pathological type & \\
Non-keratinized differentiated & $2(12.5)$ \\
Non-keratinized undifferentiated & $14(87.5)$ \\
Chemotherapy & \\
Induction & $4(25.0)$ \\
Concurrent & $14(87.5)$ \\
Adjuvant & $6(37.5)$ \\
\hline
\end{tabular}

UICC The Union of International Cancer Control, ECOG Eastern Cooperative Oncology Group

aPatients' staging status according to UICC/AJCC Cancer Staging Manual (Eighth Edition) at initial diagnosis. Two patients presented synchronous liver metastasis and were defined as stage IV, the other 14 patients presented metachronous liver metastasis and were defined as stage II or stage III according to their status of $\mathrm{T}$ criteria and $\mathrm{N}$ criteria
Table 2 Baseline of hepatic metastases $(n=16)$

\begin{tabular}{ll}
\hline Characteristics & $\mathrm{n}(\%)$ \\
\hline Hepatic involvement & \\
$\quad<25 \%$ & $3(18.8)$ \\
$25-75 \%$ & $11(68.7)$ \\
$>75 \%$ & $2(12.5)$ \\
Lobulor involvement & \\
Bilobar & $13(81.3)$ \\
Unilobar & $3(18.8)$ \\
Number of lesion & \\
$<5$ & $2(12.5)$ \\
5-9 & $5(31.3)$ \\
$>9$ & $9(56.2)$ \\
Extrahepatic metastasis & \\
No & $9(56.2)$ \\
Lung & $3(18.8)$ \\
Bone & $5(31.3)$ \\
Baseline of albumin (mg/mL) & \\
$\geq 35$ & $9(56.2)$ \\
$<35$ & $7(43.8)$ \\
Portal vein thrombus & 0 \\
Child-Pugh class & \\
A & $10(62.5)$ \\
B & $6(37.5)$ \\
\hline
\end{tabular}

\section{Response}

Intrahepatic lesions were assessed every 2 HAI cycle or when necessary, with enhanced spiral-CT scan according to RECIST criteria (version 1.1). 4 patients (25\%) achieved complete response (CR), 10 patients $(62.5 \%)$ achieved partial response (PR), 2 patients (12.5\%) achieved stable disease (SD), none with local progressive disease (PD). That is to say, the objective response rate (ORR) is $87.5 \%(\mathrm{CR}+\mathrm{PR})$, disease control rate (DCR) of intrahepatic lesions is $100 \%$ $(\mathrm{CR}+\mathrm{PR}+\mathrm{SD})$, but 3 patients $(18.8 \%)$ had extrahepatic progression during the trial treatment. See Table 3. Figure 1 demonstrates a typical CT imaging of one patient.

\section{Survival analysis}

The median follow up was 31 months (range 8 to 42 months). On May 31, 2019, 5 patients were still alive. The median overall survival (mOS) was 30 months. The mOS was 31 months and 24 months for patients with $<9$ intrahepatic lesions and $\geq 9$ lesions, respectively $(P=0.562)$. As grouped by the existence of extrahepatic metastases, patients with extrahepatic metastases had a median survival of 17 months, while patients without extrahepatic metastases had a median survival of 31 months, there was analytical significance $(P=0.005)$. See Figs. 2 and 3. 
Table 3 No. of HAI cycle, response and catheter related complications for individual patient

\begin{tabular}{lllll}
\hline Case & HAI cycles & $\begin{array}{l}\text { Intrahepatic } \\
\text { response }\end{array}$ & $\begin{array}{l}\text { Extrahepatic } \\
\text { progression }\end{array}$ & $\begin{array}{l}\text { Cather related } \\
\text { complications }\end{array}$ \\
\hline 1 & 5 & PR & No & No \\
2 & 6 & CR & No & No \\
3 & 3 & SD & Yes & No \\
4 & 8 & PR & No & No \\
5 & 3 & PR & No & Yes \\
6 & 4 & PR & No & No \\
7 & 4 & CR & No & No \\
8 & 2 & PR & Yes & No \\
9 & 6 & PR & No & No \\
10 & 2 & PR & Yes & No \\
11 & 6 & CR & No & No \\
12 & 7 & PR & No & No \\
13 & 2 & PR & No & Yes \\
14 & 4 & SD & No & No \\
15 & 6 & CR & No & No \\
16 & 4 & PR & No & No \\
\hline
\end{tabular}

\section{Side effects and adverse events}

In summary, all the 16 patients underwent a total number of 72 cycles of HAI therapy as showed in Table 3, and the number of cycles in which any side effect or adverse event could be observed or detected was accumulated. The most common toxicities include: leukopenia occur in 36 cycles (50\%) including grade $1 / 2$ in $28(38.9 \%)$ and grade $3 / 4$ in 8 (11.1\%), thrombocytopenia in $25(36.1 \%)$ including grade $1 / 2$ in $21(29.2 \%)$ and grade $3 / 4$ in $5(6.9 \%)$, AST/ALT elevation in $8(66.7 \%)$ including grade $1 / 2$ in $36(50.0 \%)$ and grade $3 / 4$ in $12(16.7 \%)$. In addition, grade $3 / 4$ abdominal pain occurred in 3 cycles (4.2). No grade 3/4 hyperbilirubinemia occurred. The main toxicities was showed by cycles as listed in Table 4. Catheter related complications occurred in 2 patients (12.5\%) including catheter occlusion in 1 patient after 2 cycles of HAI and catheter displacement occurred in 1 patient after 3 cycles (Table 3 ). HAI was discontinued in these patients. No long-term complication such as biliary toxicity and hepatic artery occlusion was observed in the study.

\section{Discussion}

Several studies have been shown that HAI is effective in improving hepatic response rates in colorectal cancer liver metastasis. In the present study, we treated 16 consecutive NPC patients with predominant liver metastasis using HAI of Gemcitabine and FUDR. Our data show that the ORR was $87.5 \%$ and the mOS was 30 months. The majority $(223 / 266,83.8 \%)$ of toxicities and adverse events was judged on Grades $1 / 2$.

About one-third of patients with NPC suffer of distant metastases and resultant treatment failure [15]. Liver metastasis is the second common metastasis site, which accounts for $30 \%$ of such cases, following bone metastasis (70\%) and followed by lung metastasis (18\%) [16], but has the worst prognosis (with reported mOS of 3-5 months) [6,7]. Thus it is of significance to explore new therapeutic strategy.

HAI has been proven both by our previous work and other authors to be helpful in some patients $[9,10]$. It is also accepted as first-line treatment for unresectable colorectal cancer liver metastases [17]. Here we administered HAI for patients with NPC liver metastasis. Given that in our case series, the majority of patients suffered of unresectable dominant liver involvement (defined as liver involvement as the dominant site of metastasis, hepatic metastases constitute $\geq 50 \%$ of all tumor burden [18]): 13 patients (81.2\%) had hepatic involvement $>25 \%, 13$ patients $(81.2 \%)$ had bilobar involvement, 14 patients (87.5\%) had number of intrahepatic lesions $>5$ (among whom 9 patients with number of intrahepatic lesions $>9$ ), the ORR and DCR of intrahepatic lesions as well as survival analysis is encouraging.

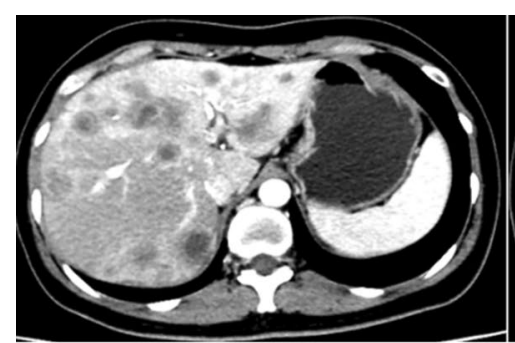

Baseline

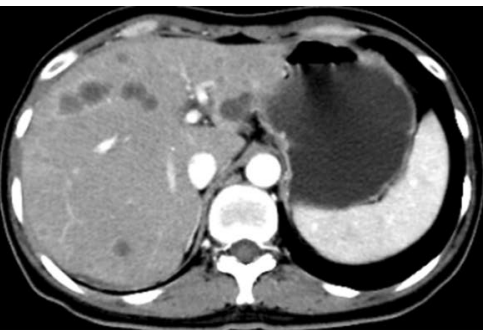

2 months

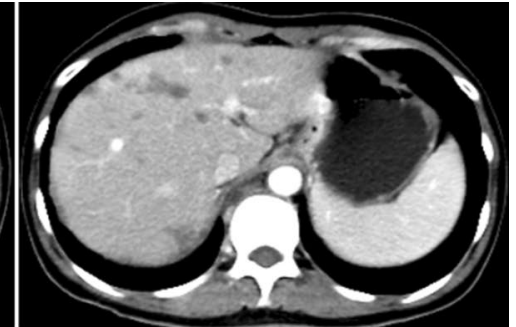

12 months
Fig. 1 A typical patient's CT imaging of liver. CT image at baseline showed extensive intra-hepatic lesions. CT image showed the intrahepatic lesions shrinking after 2 months of HAI regime. Intra-hepatic lesions further shrank after 12 months of HAI regime. This patient was judged as exhibiting a partial response 


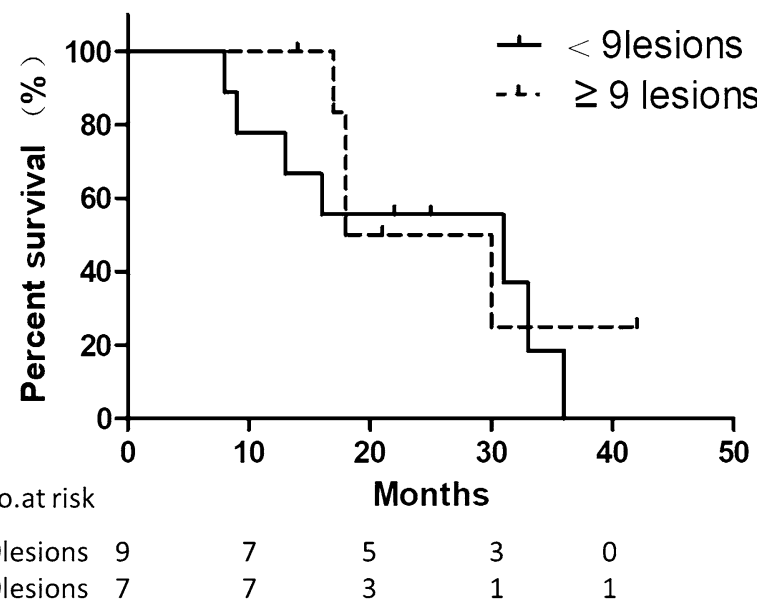

Fig. 2 Kaplan-Meier estimates of OS for patients grouped by number of intra-hepatic lesions. Median overall survival (mOS) for patients with $<9$ intra-hepatic lesions is 31 months, while mOS for patients with $\geq 9$ intra-hepatic lesions is 24 months, calculated from the date of catheter implantation. $(\mathrm{P}=0.562, \log$-rank test $)$

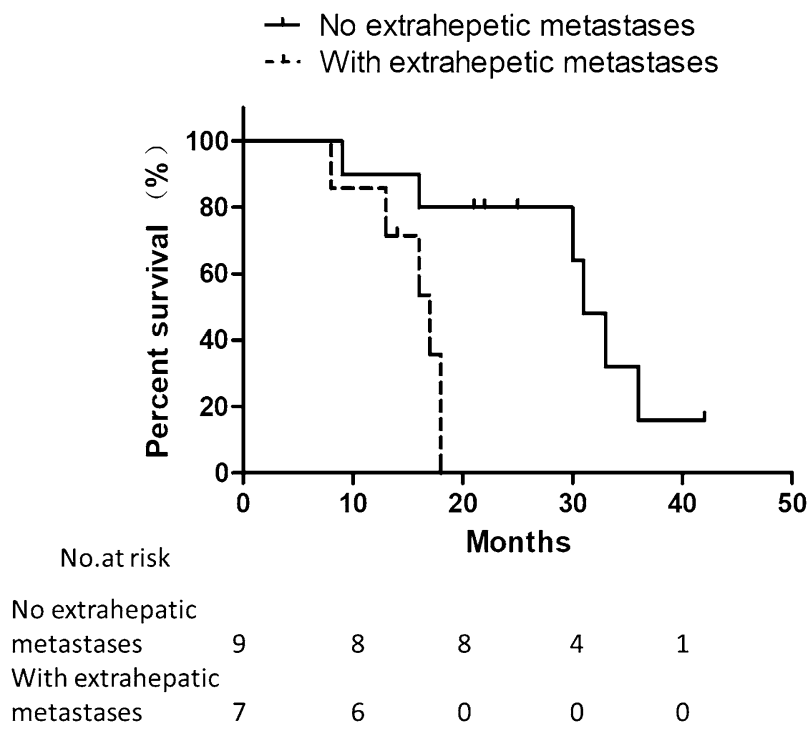

Fig. 3 Kaplan-Meier estimates of OS for patients grouped by existence of extra-hepatic metastases. Median overall survival (mOS) for patients without extra-hepatic lesions is 31 months, while mOS for patients with extra-hepatic lesions is 17 months, calculated from the date of catheter implantation. $(\mathrm{P}=0.005, \log$-rank test $)$

Nowadays treatment options for metastatic NPC include Clinical trials, Platinum-based combination chemotherapy or concurrent chemo/radiotherapy [8], but prognosis is still poor. Some investigators have reported systemic monochemotherapy for metastatic NPC with ORR range from 28 to $48 \%$ [19-21], and some polychemotherapy protocols with ORR range from 42.7 to $73 \%$ [20, 22, 23]. It seems that patients in current study probably have survival benefit.
Table 4 Most common toxicities by cycles [n (\%)]

\begin{tabular}{lll}
\hline Toxicities & $\begin{array}{l}\text { Grades } 1 / 2 \\
\mathrm{n}(\%)\end{array}$ & $\begin{array}{l}\text { Grades } 3 / 4 \\
\mathrm{n}(\%)\end{array}$ \\
\hline $\begin{array}{l}\text { Hematological } \\
\text { Leukopenia }\end{array}$ & $28(38.9)$ & $8(11.1)$ \\
Neutropenia & $26(36.1)$ & $6(8.3)$ \\
Anemia & $14(19.4)$ & $2(2.8)$ \\
Thrombocytopenia & $21(29.2)$ & $5(6.9)$ \\
AST/ALT elevation & $36(50.0)$ & $12(16.7)$ \\
Hyperbilirubinemia & $9(12.5)$ & 0 \\
Gastrointestinal & & \\
Anorexia & $8(11.1)$ & 0 \\
Nausea & $17(23.6)$ & 0 \\
Vomiting & $14(19.4)$ & $2(2.8)$ \\
$\quad$ Diarrhea & $15(20.8)$ & $3(4.2)$ \\
Fatigue & $20(27.8)$ & $2(2.8)$ \\
Abdominal pain & $15(20.8)$ & $3(4.2)$ \\
\hline
\end{tabular}

Theoretically, controlling intrahepatic lesions decreases the total tumor burden, then yields survival benefit. It is reported that elimination liver metastasis of nasopharyngeal carcinoma might improve overall survival [24], which might have something in common with current study as respect to the underlying mechanism of survival benefit.

In current study, chemotherapy agents are administered through intra-arterial approach, directly targeting intrahepatic lesions. Especially for FUDR, the low-dose and longperiod infusion may provide higher intact drug concentration in liver and minimal systemic toxicity [25].

Besides HAI, patients in current study also underwent comprehensive therapy to primary lesion. This combined treatment modality supplies control power both of the primary tumor and of the liver metastases, which may contribute to survival benefit. But there was still 3 patients (18.8\%) who had extra-hepatic progression during the trial treatment, indicating the necessity to explore more effective treatment strategies.

Several side effects and adverse events could be observed or detected in current study, including (i) hematological toxicities such as leukopenia, neutropenia, anemia, thrombocytopenia; (ii) AST/ALT elevation; (iii) hyperbilirubinemia; (iv) gastrointestinal toxicities such as anorexia, nausea, vomiting, diarrhoea; (v) fatigue; (vi) abdominal pain. For all the toxicities mentioned above, the proportion for grades $1 / 2$ toxicities is obviously higher than that for grades $3 / 4$ toxicities. Some toxicities resulted from the same underlying mechanism as systemic chemotherapy. AST/ALT elevation appeared in 48 cycles (66.7\%), but the majority of them $(n=36,50 \%)$ was on grades $1 / 2$, while the minority $(n=12,16.7 \%)$ was on grades $3 / 4$. This might reveal the safety of this strategy for liver function. Some authors 
reported biliary toxicity of HAI with FUDR [25]. In current study, reversible Grade1/2 Hyperbilirubinemia is observes in $9(12.5 \%)$ cycles, with no evidence of long-term biliary damage, probably because of adding DXM into FUDR solution. Technically, the gastrointestinal toxicities might derive from extra-hepatic infusion of FUDR to stomach or duodenum, so the main branch vessels from the associated arteries (e.g. Hepatic artery or GDA) were routinely embolized in operation, but this still could not be completely avoided. Fortunately, most patients with grades $3 / 4$ toxicities continued HAI therapy, even though some needed dose reduction or gap prolongation. If indwelled directly in hepatic artery, the dislodgement of the infusion catheter tip may result in vessel damage, occlusion or aneurysm, finally lead to failure of the whole infusion system. Here we fixed the side-hole infusion catheter in GDA or in the relatively smaller branch of hepatic artery, positioned the side-hole in common hepatic artery, thus effectively avoided the potential risk and guaranteed the unidirectional infusion to liver. Other HAI related complications, such as bleeding, thrombosis or infection are not observed in these patients. In our opinion, these data, to some extent, could prove the safety of our HAI strategy.

This study has several limitations. We performed HAI for NPC patients with liver-predominant metastasis, whose intrahepatic disease status is more advanced compared to those who do not undergo HAI. As a retrospective study with small sample size, it lacks a control group with matched status of NPC liver metastases. The improvement of DCR of intra-hepatic lesions and survival benefit needs to be further investigated and confirmed by multi-center randomized study.

In conclusion, we introduced a new treatment strategy for NPC liver metastases, which may improve DCR of intrahepatic lesions and prolong mOS. It can be considered as an option for suitable patients.

Open Access This article is licensed under a Creative Commons Attribution 4.0 International License, which permits use, sharing, adaptation, distribution and reproduction in any medium or format, as long as you give appropriate credit to the original author(s) and the source, provide a link to the Creative Commons licence, and indicate if changes were made. The images or other third party material in this article are included in the article's Creative Commons licence, unless indicated otherwise in a credit line to the material. If material is not included in the article's Creative Commons licence and your intended use is not permitted by statutory regulation or exceeds the permitted use, you will need to obtain permission directly from the copyright holder. To view a copy of this licence, visit http://creativecommons.org/licenses/by/4.0/.

\section{References}

1. Si Y, Lan G, Deng Z et al (2016) Distribution and clinical significance of circulating tumor cells in nasopharyngeal carcinoma. Jpn J Clin Oncol 46(7):622-630
2. Wei KR, Zheng RS, Zhang SW, Liang ZH, Ou ZX, Chen WQ (2014) Nasopharyngeal carcinoma incidence and mortality in China in 2010. Chin J Cancer 33(8):381-387

3. Tu Z, Chen Q, Zhang JT, Jiang X, Xia Y, Chan HC (2016) CFTR is a potential marker for nasopharyngeal carcinoma prognosis and metastasis. Oncotarget 7(47):76955-76965

4. Chan AT (2010) Nasopharyngeal carcinomaa. Ann Oncol. 21:vii308-vii312

5. Siti-Azrin AH, Norsa'adah B, Naing NN (2017) Prognostic factors of nasopharyngeal carcinoma patients in a tertiary referral hospital: a retrospective cohort study. BMC Res Notes 10(1):705

6. Pan C, Tao Y, Zhao M et al (2012) Comparative serum proteomic analysis involving liver organ-specific metastasis-associated proteins of nasopharyngeal carcinoma. Exp Ther Med 3(6):1055-1061

7. Tomizawa N, Ohwada S, Ogawa $T$ et al (2006) Factors affecting the prognosis of anatomical liver resection for liver metastases from colorectal cancer. Hepatogastroenterology 53(67):89-93

8. NCCN Clinical Practice Guidelines in Head and Neck Cancers (2018 Version I) [DB/OL]. https://www.nccn.org

9. Groot KB, Sadot E, Kemeny NE et al (2017) Perioperative hepatic arterial infusion pump chemotherapy is associated with longer survival after resection of colorectal liver metastases: a propensity score analysis. J Clin Oncol 35(17):1938-1944

10. Shi L, Zhao J, Lu Q et al (2015) Initial hepatic artery infusion and systemic chemotherapy for asymptomatic colorectal cancer with un-resectable liver metastasis. Int J Clin Exp Med 8(1):1000-1008

11. NCCN Clinical Practice Guidelines in Neuroendocrine Tumors (2017 Version I) [DB/OL]. https://www.ncen.org

12. Herrmann KA, Waggershauser T, Sittek H, Reiser MF (2000) Liver intraarterial chemotherapy: use of the femoral artery for percutaneous implantation of catheter-port systems. Radiology 215(1):294-299

13. Tanaka T, Arai Y, Inaba Y et al (2003) Radiologic placement of side-hole catheter with tip fixation for hepatic arterial infusion chemotherapy. J Vasc Interv Radiol 14(1):63-68

14. Eisenhauer EA, Therasse P, Bogaerts J et al (2009) New response evaluation criteria in solid tumours: revised RECIST guideline (version 1.1). Eur J Cancer. 45(2):228-247

15. Hong S, Zhang L (2016) Gemcitabine improves survival in patients with recurrent or metastatic nasopharyngeal carcinoma. Chin J Cancer 35(1): 100

16. Ahmad A, Stefani S (1986) Distant metastases of nasopharyngeal carcinoma: a study of 256 male patients. J Surg Oncol 33(3):194-197

17. Karanicolas PJ, Metrakos $P$, Chan K et al (2014) Hepatic arterial infusion pump chemotherapy in the management of colorectal liver metastases: expert consensus statement. Curr Oncol 21(1):e129-e136

18. Tsimberidou AM, Moulder S, Fu S et al (2010) Phase I clinical trial of hepatic arterial infusion of cisplatin in combination with intravenous liposomal doxorubicin in patients with advanced cancer and dominant liver involvement. Cancer Chemother Pharmacol 66(6):1087-1093

19. Foo KF, Tan EH, Leong SS et al (2002) Gemcitabine in metastatic nasopharyngeal carcinoma of the undifferentiated type. Ann Oncol 13(1):150-156

20. Ma BB, Tannock IF, Pond GR, Edmonds MR, Siu LL (2002) Chemotherapy with gemcitabine-containing regimens for locally recurrent or metastatic nasopharyngeal carcinoma. Cancer 95(12):2516-2523

21. Zhang L, Zhang Y, Huang PY, Xu F, Peng PJ, Guan ZZ (2008) Phase II clinical study of gemcitabine in the treatment of patients with advanced nasopharyngeal carcinoma after the 
failure of platinum-based chemotherapy. Cancer Chemother Pharmacol 61(1):33-38

22. Ngan RK, Yiu HH, Lau WH et al (2002) Combination gemcitabine and cisplatin chemotherapy for metastatic or recurrent nasopharyngeal carcinoma: report of a phase II study. Ann Oncol 13(8):1252-1258

23. Wang J, Li J, Hong X et al (2008) Retrospective case series of gemcitabine plus cisplatin in the treatment of recurrent and metastatic nasopharyngeal carcinoma. Oral Oncol 44(5):464-470

24. Ruo-Han Tseng HW, Cheng-Hui Chung EA (2017) Elimination liver metastasis of nasopharyngeal carcinoma might improve overall survival. J Cancer Res Pract 4:63-65
25. Aldrighetti L, Arru M, Ronzoni M, Salvioni M, Villa E, Ferla G (2001) Extrahepatic biliary stenoses after hepatic arterial infusion (HAI) of floxuridine (FUdR) for liver metastases from colorectal cancer. Hepatogastroenterology 48(41):1302-1307

Publisher's Note Springer Nature remains neutral with regard to jurisdictional claims in published maps and institutional affiliations. 